UCRL-JC-103920

PREPRINT

\title{
Dielectronic Recombination Into Rydberg Levels of Lithiumlike Titanium
}

\author{
P. Beiersdorfer \\ S. Chantrenne \\ M. H. Chen \\ R. E. Marrs \\ D. A. Vogel \\ K. L. Wong \\ R. Zasadzinski \\ High Temperature Physics Division \\ Prepared for the Submittal to \\ Zeitschrilt f. Physik D \\ $\mathrm{V}^{\text {th }}$ International Conference on \\ the Physics of Highly-Charged Ions. \\ Giessen, FRG
}

September 10-14, 1990

October 10, 1990

This is a preprint of a paper intended for publication in a journal or proceedings. Since changes may be made before publication, this preprint is made available with the understanding that it will not be cited or reproduced without the permission of the author. 


\section{DISCIAINIKR}

This document was prepared as an account of work sponsored by an agenc! of the I'nited States Government. Neither the linited States Government nor the l nisersity of California nor ans of their employees, makes any narrants. ¿..press or implied, or assumes any legal liability or responsibility for the accuracy, completeness, or usefulness of an! information, apparatus, product. or process disclosed, or represents that its use would not infringe prisatels ouned rights. Reference herein to an! specific commercial products, precess, or service by tradi naitle, trademark, manufacturer, or otheruise. does not necessurily constitute or imply its aldorsement, recommendation. or favoring by the Enited States Ciesernment or sthe Lniversity of California. The viens and opinions of authors espressed herein do not necessarily state or reflect those of the I'nited States (jovernment or the University of (alifornis. and shall not he used for advertising or product endorsement purposes. 


\title{
Dielectronic Recombination Into Rydberg Levels of Lithiumlike Titanium
}

P. Beiersdorfer, S. Chantrenne, M. H. Chen, R. E. Marrs, D.

A. Vogel, K. L. Wong, and R. Zasadzinski

High Temperature Physics Division, University of California, Lawrence Livermore National

Laboratory, Livermore, CA 94550

\begin{abstract}
To characterize line formation near threshold for electron-impact excitation, dielectronic capture into high- $n$ levels in lithiumlike $\mathrm{Ti}^{19+}$ ions has been studied with high-resolution $x$-ray spectroscopy on the Livermore electron beam ion trap. The process is shown to result in satellite lines which are virtually indistinguishable from their dipole-allowed heliumlike parent lines. High- $n$ satellite lines are absent from dipole-forbidden heliumlike lines.
\end{abstract}




\section{Introduction}

Dielectronic capture by heliumlike ions into levels with high principal quantum number $n$,

$$
1 s^{2}+e^{-} \rightarrow 1 s 2 \ln l^{\prime}
$$

results in $x$ rays

$$
1 s 2 \ln l^{\prime} \rightarrow 1 s^{2} n l^{\prime}+h \nu_{1}
$$

with energy very close to that of the heliumlike parent line

$$
1 s 2 l \rightarrow 1 s^{2}+h \nu_{2}
$$

In plasma, dielectronic capture and electron-impact excitation processes occur concurrently, and $x$ rays enitted in the radiative stabilization blend with the parent line and form sateilite lines on the low-energy side, which typically cannot be resolved [1-3].

We have studied the process of high-n dielectronic capture by heliumlike $\mathrm{Ti}^{20+}$ ions with the Li'vermore $\mathrm{EBIT}$ [4], which probes ions with a nearmonoenergetic electron beam. If the beam energy is set to a value just below the threshold for direst excitation, dielectronic capture into high- $n$ levels ensues, and $x$ rays resulting from subsequent radiative stabilization can be observed in the absece of their parent lines.

The measurements show that only the two dipole-allowed transitions $w$ and $y$, which proceed from the upper levels $1 s 2 p^{1} P_{1}$ and $1 s 2 p{ }^{3} P_{1}$, respectively, are associated with high- $n$ satellites lines. The magnetic quadrupole transition $x$ and the magnetic dipole transition $z$, which proceed from the upper levels $1 s 2 p{ }^{3} P_{2}$ and $1 s 2 s{ }^{3} S_{1}$, respectively, are not accompanied by high- $n$ satellites. This behavior is consistent with the relative magnitude of the radiative decay rates of the heliumlike transitions and of the Auger rates of the high- $n$ doubly excited levels. 


\section{Experiment}

The $n=2$ to $n=1$ transitions of $\mathrm{Ti}^{20+}$ are measured using the EBIT high-resolution von Hámos spectrometer [5], which observes x rays in a plane perpendicular to the direction of the electron beam. The spectrometer employs a $50-\mathrm{mm} \times 25-\mathrm{mm} \times 0.25-\mathrm{mm} \mathrm{LiF}$ crystal bent to a radius of curvature of $30 \mathrm{~cm}$. The crystal is cut parallel to the (200) plane with a $2 \mathrm{~d}$-spacing of $4.027 \AA$. Consequently, the heliumlike transitions are observed at a near $45^{\circ}$ Bragg angle. At this angle, the crystal acts as a polarizer, and detected $\mathrm{x}$ rays are polarized parallel to the beam direction [6]. A helical delay line proportional counter [7] with a sensitive area of $3 \times 11 \mathrm{~cm}^{2}$ is employed to record the spectra. The resolving power of the spectrometer is about $\lambda / \Delta \lambda=1000$, which is sufficient to resolve all directly excited heliumlike lines.

Accurate knowledge of the beam-ion interaction energy is important for our measurement. Because of the space charge of the electron beam the interaction energy is not simply determined from the acceleration potential. Instead, we have determined the interaction energy to within $10 \mathrm{eV}$ by measuring the energy of the photons emitted in the radiative capture of beam electrons by bare and hydrogenlike argon ions with a solid-state detector. Such emission is always present for beam energies above about $4 \mathrm{keV}$, as small quantities of argon are part of the residual gas.

$\mathrm{K}$ x-ray spectra of heliumlike $\mathrm{Ti}^{20+}$ obtained at three different beam energies are shown in Fig. 1. The spectrum in (a) is obtained at a beam energy of $4986 \mathrm{eV}$, which is well above the direct electron-impact excitation threshold for all four heliumlike transitions (cf. Table I), and all four heliumlike lines are seen. The spectrum in (b) is obtained at $4685 \mathrm{eV}$, which is $49 \mathrm{eV}$ below the threshold for $x$. Indeed, $x$ is not seen in the spectrum. On the other hand, $z$ and lines that appear to be $w$ and $y$ continue to be present. 
$z$ has disappeared in (c), which was recorded $53 \mathrm{eV}$ below its threshold; apparent lines $w$ and $y$, however, persist in the spectrum. The latter lines are formed by dielectronic capture into Rydberg levels and subsequent radiative stabilization. Because of a close match of their transition energies, they are virtually indistinguishable from their parent transitions. The spectra also show the two principal lithiumlike lines $q$ and $r$, i.e., the transitions $1 s 2 s 2 p^{2} P_{3 / 2} \rightarrow 1 s^{2} 2 s^{2} S_{1 / 2}$ and $1 s 2 s 2 p^{2} P_{1 / 2} \rightarrow 1 s^{2} 2 s^{2} S_{1 / 2}$. Below threshold for direct excitation, apparent lines $q$ and $r$ are formed dielectronically like the apparent heliumlike lines.

The relative line intensities of the heliumlike lines and their associated high- $n$ satellite lines are shown in Fig. 2 as a function of beam energy in the range $4.6 \leq E_{\text {beam }} \leq 5.1 \mathrm{keV}$. All intensities are normalized to $w$. The figure demonstrates that $x$ and $z$ vanish about $50 \mathrm{eV}$ below threshold; no apparent lines due to high- $n$ satellites form below threshold that extend their excitation functions. Indeed, the onset of $x$ and $z$, as the threshold is crossed, is consistent with a 50-eV FWHM spread in the energy distribution of the electron beam measured earlier with a K-edge filter by Levine et al. [8] This is not true for $y$ and, implicitly, for $w$. Their excitation functions do not vanish below threshold, but smoothly match those of their apparent lines, as indicated by Fig. 2(c).

The high- $n$ spectator electron shifts the transition energies of apparent lines slightly from those of their heliumlike parent lines. We have measured the shift in case of apparent line $w$, and results are plotted in Fig. 3. All shifts are toward lower enersy. In a plasma, where high- $n$ satellites blend with their parent line, this casuses a redshift and broadening of $w$. As a result, the presence of high- $n$ satellites can affect determinations of ion temperature from the line width and of plasma streaming from the line position, 
as demonstrated by Bitter et al. [3] In addition, their presence limits the accuracy with which transition energies can be measured [9].

To complete the characterization of the behavior of the heliumlike lines near threshold, our measurements extend to the KMM dielectronic resonance centered at $E_{B e a m}=4.95 \mathrm{keV}$. Here resonant capture of a beam electron substantially enhances emission of the heliumlike lines via the process

$$
1 s^{2}+e^{-} \rightarrow 1 s 3 l 3^{\prime} \rightarrow 1 s 2 l+e^{-} .
$$

\section{Discussion}

The marked differences in the threshold behavior of the four heliumlike lines can be explained with $\Rightarrow$ look at their respective radiative rates and the Auger rates of the associated high- $n$ configurations.

The satellite intensity is proportional to the so-called line factor $[10,11]$,

$$
F_{2}=\frac{g_{s}}{g_{i}} \frac{A_{a}^{s i} A_{r}^{s f}}{\Sigma_{j} A_{a}^{s j}+\Sigma_{f^{\prime}} A_{r}^{s f^{\prime}}} .
$$

$A_{a}^{s i}$ is the autoionization rate for decay of upper level $|s\rangle$ to the ground state $|i\rangle$ of the recombing ion; $A_{r}^{s f}$ is the rate of radiative decay of upper level $|s\rangle$ to lower level $|f\rangle ; g_{s}$ and $g_{i}$ are the statistical weights of the autoionizing level and of the ground state of the recombining ion, respectively. The sum over $f^{\prime}$ extends over all levels lower than $|s\rangle$; the sum over $|j\rangle$ extends over all levels that are populated by autoionization of $|s\rangle . A_{r}^{s f}$ can be approximated by the radiative rate of the associated parent line (cf. Table I), while $A_{a}^{s i} \approx 10^{+13}$ $\mathrm{s}^{-1}$ for $n=4$ and drops as $n^{-3}$. Hence, line factors of high- $n$ satellites to the heliumlike electric dipole lines are as large as $F_{2} \approx 10^{+13} \mathrm{~s}^{-1}$; those to the magnetic dipole and quadrupole lines have lines factors of only $F_{2} \leq 10^{10} \mathrm{~s}^{-1}$. Consequently, dielectronic capture into Rydberg levels is a strong process 
only for dipole-allowed parent transitions with large radiative rates, and their intensity matches smoothly with the intensity of their high-n satellites, as the threshold for direct excitation is crossed. By contrast, dipole-forbidden lines are devoid of matching high-n satellites as a result of the competition between autoionization and radiative stabilization of high- $n$, doubly excited levels, and turn on in a step-like fashion.

The differences in threshold behavior of dipole-forbidden and dipoleallowed transitions suggests a means for identifying contributions from different transitions in blends. In heliumlike ions that have a finite nuclear moment the $1 s 2 p^{3} P_{0}$ level decays to the ground state via the hyperfine interaction and blends with $y$. Its radiative decay rate is about $10^{+10} \mathrm{~s}^{-1}$ for heliumlike ions of the transition metals [12]. Like $x$ the line is, therefore, unaffected by high-n satellite transitions and should turn on in a step-like fashion as the direct excitation threshold is crossed. This step should be a distinct feature in the excitation function of $y$. Experiments involving $\mathrm{V}^{21+}$ or $\mathrm{Mn}^{23+}$ are planned to confirm these expectations. The dominant isotope of Ti has a vanishing nuclear moment, and no such step is seen in Fig. 2(c).

\section{Acknowledgments}

We thank D. Nelson, E. Magee, and A. Hinz for their technical support. This work was performed under the auspices of the U. S. Department of Energy by Lawrence Livermore National Laboratory under contract No. W7405-ENG-48. 


\section{References}

[1] F. Bely-Dubau, A. H. Gabriel, and S. Volonté, Mon. Not. R. Astron. Soc. 189, 801 (1979).

[2] F. Bely-Dubau, P. Faucher, L. Steenman-Clark, M. Bitter, S. von Goeler, K. W. Hill, C. Cmahy-Val, and J. Dubau, Phys. Rev. A 26 (1982) 3459.

[3] M. Bitter, K. W. Hill, M. Zarnstorf, S. von Goeler, R. Hulse, L. C. Johnson, N. R. Sauthoff, S. Sesnic, and K. M. Young, Phys. Rev. A 32 , 3011 (1985).

[4] M. A. Levine, R. E. Marrs, J. R. Henderson, D. A. Knapp, and M. B. Schneider, Phys. Scr. T22, 157 (1988); R. E. Marrs, M. A. Levine, D. A. Knapp, and J. R. Henderson, Phys. Rev. Lett. 60, 1715 (1988).

[5] P. Beiersdorfer, R. E. Marrs, J. R. Herderson, D. A. Knapp, M. A. Levine, D. B. Platt, M. B. Schneider, D. A. Vogel, and K. L. Wong, Rev. Sci. Instrum. 61, (1990).

[6] J. R. Henderson, P. Beiersdorfer, C. L. Bennett, S. Chantrenne, D. A. Knapp, R. E. Marrs, M. B. Schneider, K. L. Wong, G. A. Doschek, J. F. Seely, C. M. Brown, R. E. LaVilla, J. Dubau, and M. A. Levine, Phys. Rev. Lett. 65, 705 (1990).

[7] E. Källne, J. Källne, L. G. Atencio, J. Chmielewski, G. Idzorek, and C. L. Morris, Rev. Sci. Instrum. 58 (1987) 1077.

[8] M. A. Levine, R. E. Marrs, J. N. Bardsley, P. Beiersdorfer, C. L. Bennett, M. H. Chen, T. Cowan, D. Dietrich, J. R. Henderson, D. A. Knapp, A. Osterheld, B. M. Penetrante, M. B. Schneider, and J. H. Scofield, Nucl. Instrum. Methods B43 (1989) 431. 
[9] P. Beiersdorfer, M. Bitter, S. von Goeler, and K. W. Hill, Phys. Rev. A 40 (1989) 150.

[10] A. H. Gabriel, Mon. Not. R. Astron. Soc. 160, 99 (1972).

[11] J. Dubau and S. Volonté, Rep. Prog. Phys. 43 (1980) 199.

[12] P. J. Mohr, in Beam-Foil Spectroscopy, edited by I. A. Sellin and D. J. Pegg (Plenum Press, New York, 1976), Vol. I, p. 97.

[13] C. D. Lin, W. R. Johnson, and A. Dalgarno, Phys. Rev. A 15 (1977) 154.

[14] G. W. F. Drake, Can. J. Phys. 66 (1988) 586. 


\section{Tables}

TABLE I. Radiative rates (Ref. 13) and threshold energies for direct electronimpact excitation (Ref. 14) of $w, x, y$, and $z$ in $\mathrm{Ti}^{20+}$.

\begin{tabular}{ccc}
\hline \hline Line & $A_{r}\left(\mathrm{~s}^{-1}\right)$ & $E_{t h}(\mathrm{eV})$ \\
\hline$w$ & $2.41 \times 10^{14}$ & 4749.6 \\
$x$ & $1.66 \times 10^{9}$ & 4733.8 \\
$y$ & $1.06 \times 10^{13}$ & 4726.9 \\
$z$ & $3.76 \times 10^{7}$ & 4702.0 \\
\hline
\end{tabular}




\section{Figures}

Fig. 1. K x-ray spectra of $\mathrm{Ti}^{20+}$ obtained at beam energies (a) $4985 \mathrm{eV}$, (b) $4685 \mathrm{eV}$, and (c) $4649 \mathrm{eV}$. Lines labeled in quotation marks are excited by dielectronic capture of beam electrons into high- $n$ levels.

Fig. 2. Relative line intensities of the forbidden and intercombination lines in $\mathrm{Ti}^{20+} . \mathrm{E}_{t h}$ denotes the threshold energy for electron-impact excitation. The enhancement at $4.95 \mathrm{keV}$ is due to resonance excitation.

Fig. 3. Shift of apparent line $w$ below threshold for electron-impact excitation $\left(\mathrm{E}_{t h}\right)$. Measurements are relative to the position of $w$ above threshold. 

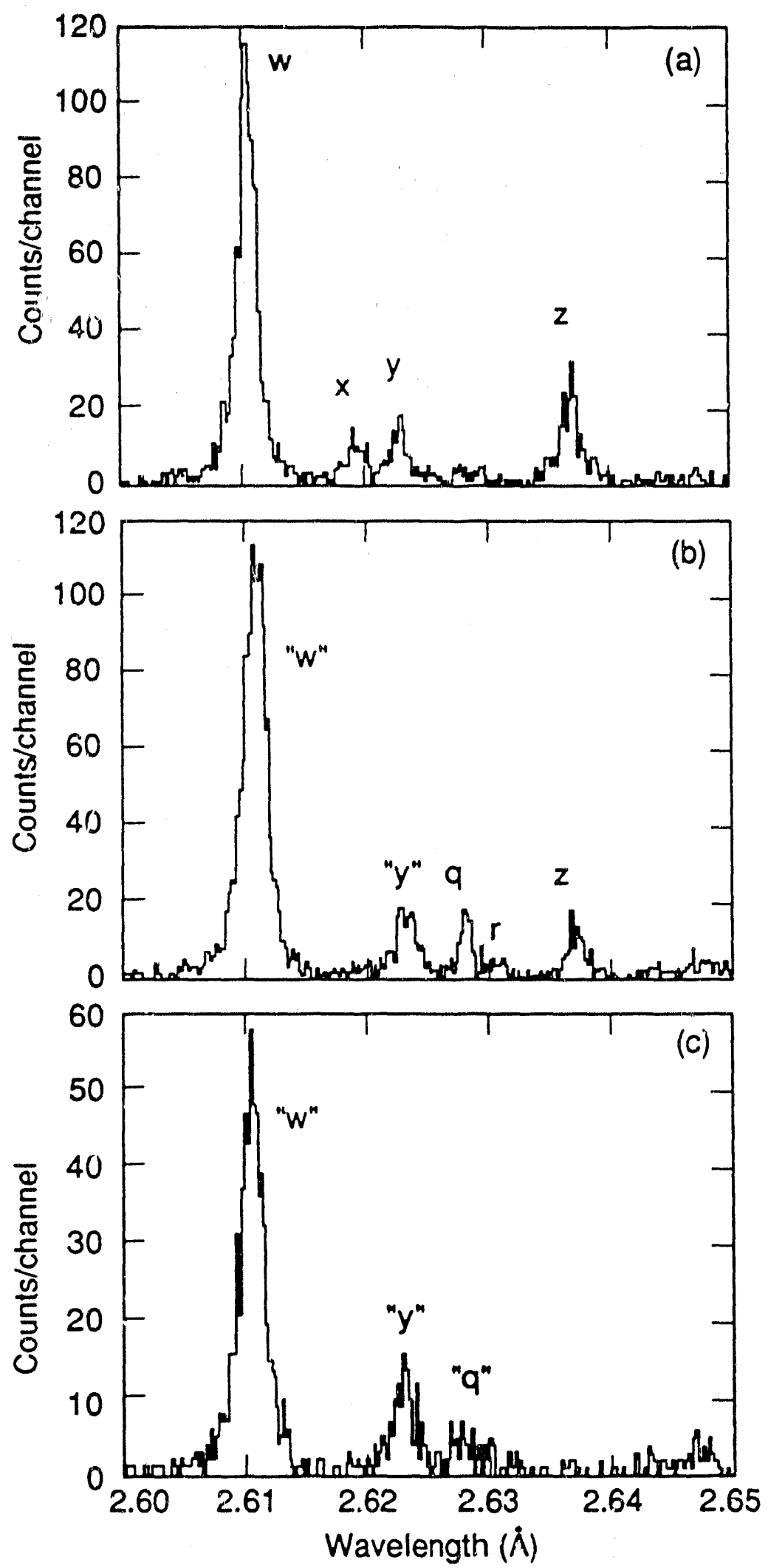

Figure 1. 


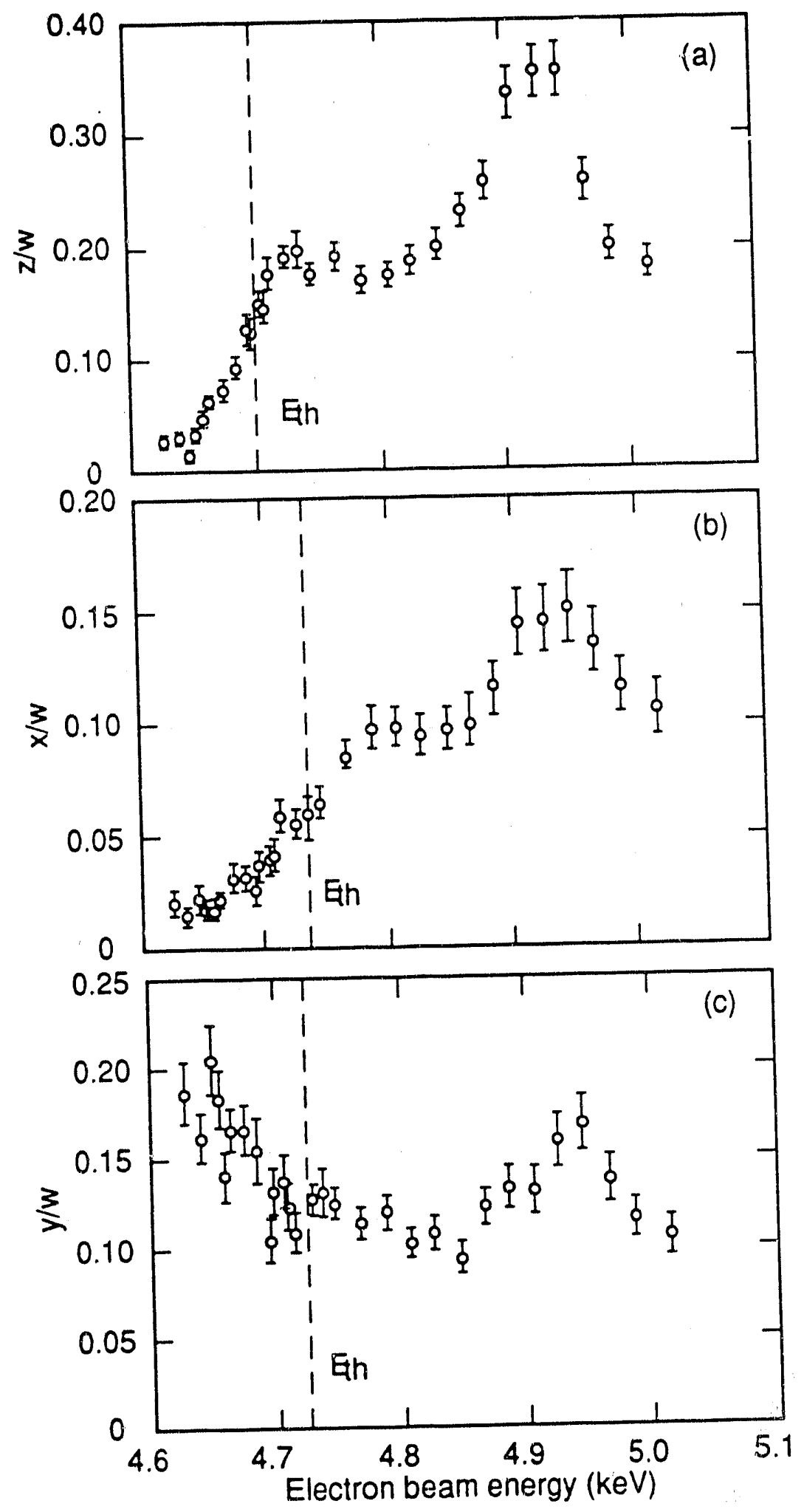

Figure 2. 


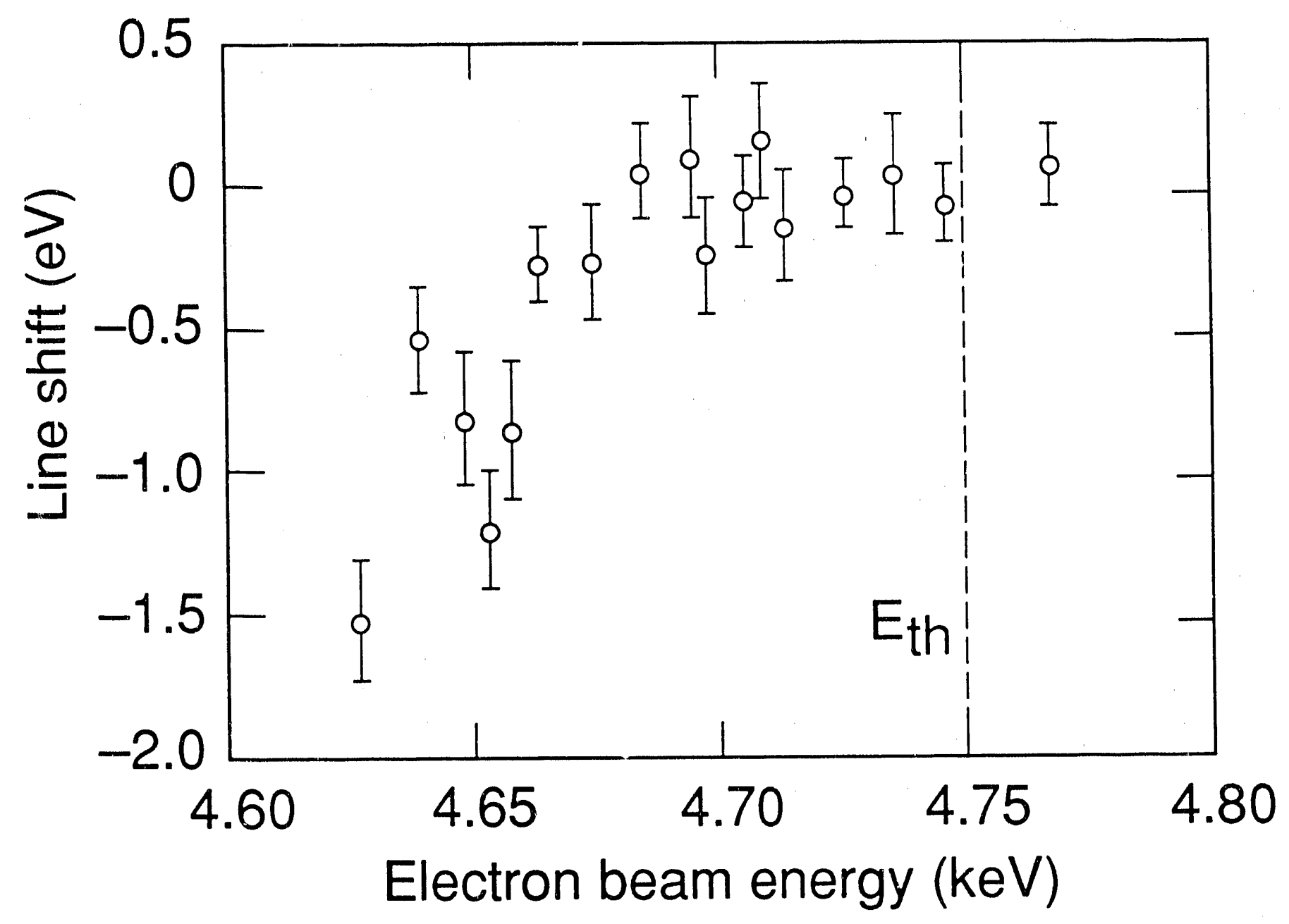

Figure 3. 

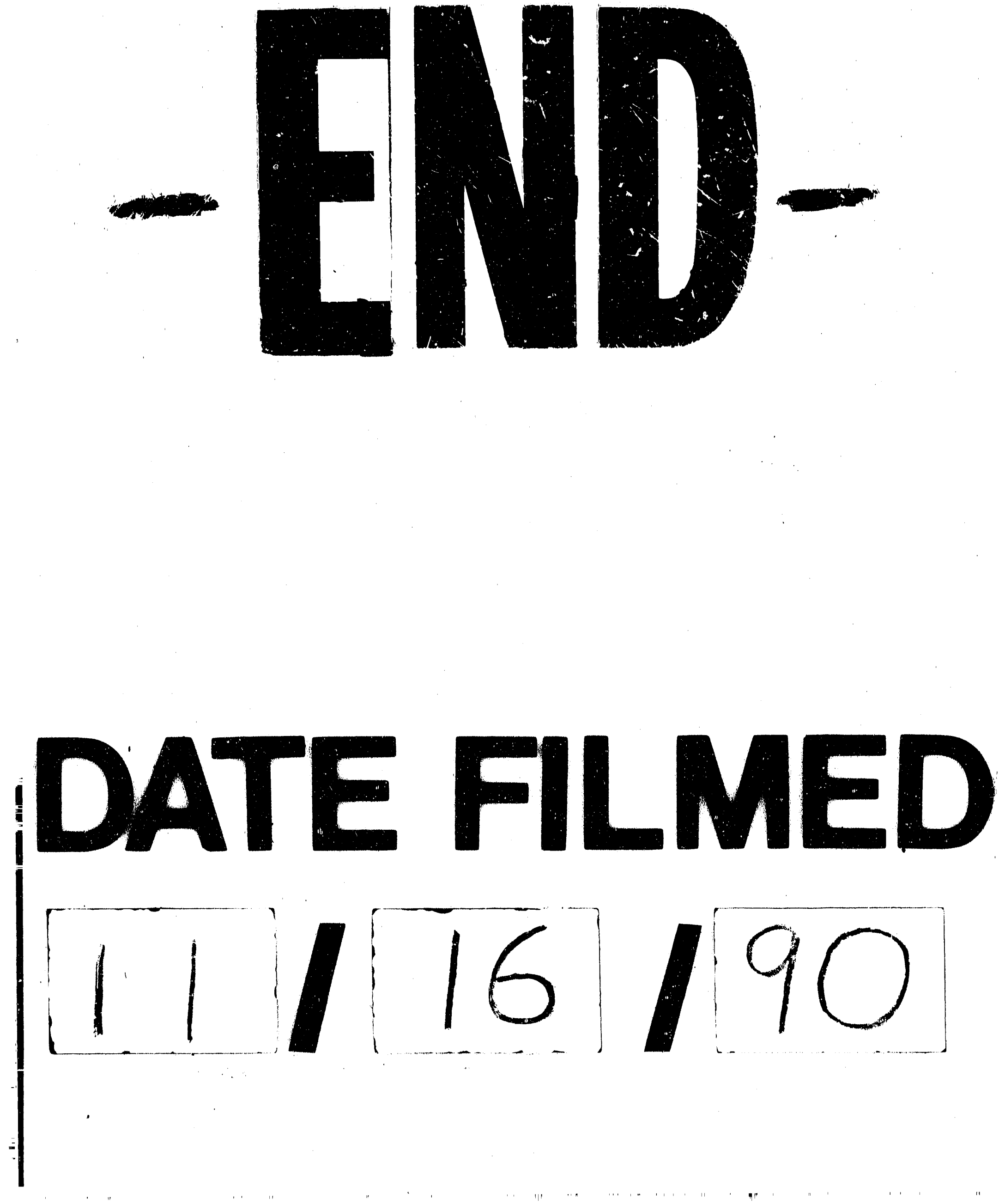
1 